\title{
Madres adolescentes internadas junto con sus hijos en un hospital de alta complejidad: diferencias entre la adolescencia temprana-media y tardía Adolescent mothers admitted with their children in a highly complex hospital: differences between early-middle and late adolescence
}

\author{
Dra. Valeria Bulgach , Dra. Cecilia Zunana ${ }^{b}$, Dra. Paula Califano ${ }^{b}$, \\ Dra. M. Susana Rodríguez y Dr. Roberto Mato ${ }^{d}$
}

\section{RESUMEN}

La maternidad adolescentees de alta prevalencia. Objetivos. Describir las características de las madres adolescentes con hijos internados en un hospital pediátrico dealta complejidad y explorar las diferencias entre la adolescencia tempranamedia y tardía.

Método. Estudio observacional y transversal en madres de 10 a 19 años y 11 meses. Se utilizó una encuesta con variables sociodemográficas de las madres, datos del embarazo, parto y recién nacido.

Resultados. Se incluyeron 227 madres, $100<17$ años y $127 \geq 17$ años. En ambos grupos etarios, un $30 \%$ y $33 \%$ tuvieron hijos prematuros, $12 \%$ y $2 \%$ tuvieron niños con muy bajo peso de nacimiento, respectivamente. Más de las dos terceras partes de las adolescentes eran hijas de madres adolescentes $-76(76 \%)$ vs. $77(61 \%)$-.

Conclusiones. Ambos grupos presentaron alta tasa de prematurez. Las más jóvenes tuvieron más niños con muy bajo peso de nacimiento. La historia intergeneracional de embarazo adolescente se repitió en más de dos tercios de las adolescentes.

Palabras clave: adolescente, madres, poblaciones vulnerables, prevención.

a. Equipo de Adolescencia.

b. Ginecología.

c. Cuidados Intermedios y Moderados.

d. Clínica de Adolescencia.

Hospital de Pediatría SAMIC "Prof. Dr. Juan Pedro Garrahan", Ciudad de Buenos Aires.

Correspondencia: Dra. Valeria Bulgach: valebulg@hotmail.com

Financiamiento:

Ninguno.

Conflicto de intereses: Ninguno que declarar.

Recibido: 10-5-2017

Aceptado: 14-12-2017 http:/ / dx.doi.org/10.5546/ aap.2018.160

Cómo citar: Bulgach V, Zunana C, Califano P, et al Madres adolescentes internadas junto con sus hijos en un hospital de alta complejidad: diferencias entre la adolescencia temprana-media y tardía. Arch Argent Pediatr 2018;116(2):160-164.

\section{INTRODUCCIÓN}

La adolescencia se define como la etapa que transcurre entre los $10 \mathrm{y}$ los 19 años. ${ }^{1}$

El embarazo durante esta etapa es un fenómeno de alta prevalencia; en Argentina, cerca del 15\% de los partos registrados por año son de una madre adolescente, tendencia que se mantiene estable desde hace más de una década. En 2015, nacieron 770040 niños; 111699 eran de madres adolescentes. $^{2}$

El embarazo adolescente, en la mayoría de los casos, no suele ser el resultado de una decisión deliberada, sino, más bien, de la ausencia de opciones. Es un acontecimiento en el que converge la falta de información sobre la sexualidad, el desconocimiento del propio cuerpo y de las posibilidades de la anticoncepción y la existencia de mitos y mandatos culturales. Estos factores configuran formas de vulneración de los derechos, generalmente, en contextos familiares adversos. ${ }^{3}$ Otro factor no menos importante es la falta de abordaje en salud adecuado, en el que se generan barreras al acceso, determinadas por el sistema y por construcciones culturales. ${ }^{4,5}$ Es por eso por lo que se recomienda que la atención de estos pacientes sea en un espacio amigable con un enfoque integral equitativo, accesible y apropiado. 
Algunas investigaciones enfocan el embarazo adolescente desde el punto de vista etario como un grupo homogéneo de riesgo. Sin embargo, es esperable un comportamiento diferente en las primeras etapas vecinas a la niñez (adolescencia temprana-intermedia, de 10 a 16 años y 11 meses) que en las etapas finales cuando se alcanza la edad adulta. ${ }^{6}$

El Hospital de Pediatría SAMIC "Prof. Dr. Juan P Garrahan" es un hospital de alta complejidad que se encuentra en la Ciudad de Buenos Aires y presenta, aproximadamente, 26000 egresos por año. Entre el 5\% y el 6\% de esos egresos corresponden a hijos de madres adolescentes. En valores absolutos, alrededor de 1500 madres adolescentes permanecerán internadas con sus hijos por año. Esta realidad nos enfrentó a la necesidad de conocer a estas madres adolescentes, que realizaron sus controles de embarazo fuera del Hospital, y poder comparar variables biológicas, perinatales y psicosociales. Asimismo, se plantea no perder oportunidades de intervención y mejorar la calidad de la atención de la díada hijo/madre adolescente.

\section{OBJETIVOS}

1. Describir las características de las madres adolescentes cuyos hijos se encuentran internados en un hospital pediátrico de alta complejidad.

2. Explorar las diferencias entre las madres en la adolescencia temprana-media ( $<17$ años) y la adolescencia tardía ( $\geq 17$ años).

\section{POBLACIÓN}

Madres adolescentes internadas junto con sus hijos menores de 1 año en alguna de las salas de un hospital pediátrico de alta complejidad.

\section{MÉTODOS}

Se realizó un estudio observacional, transversal de madres adolescentes. Las madres fueron detectadas a través de un alerta informático que se generaba durante la internación. Se seleccionó una muestra por conveniencia, que incluyó a aquellas madres internadas que se encontraban disponibles cuando el profesional se acercó a realizar la encuesta y que se encontraban en los 3 sectores de internación más accesibles a los investigadores.

Se utilizó una encuesta confeccionada por los autores a tales fines, que incluía variables demográficas, sociales y familiares de las madres, características y finalización del embarazo y puerperio, edad gestacional y peso de nacimiento de los recién nacidos ( $\mathrm{RN}$ ) (véase Anexo en formato electrónico).

Esta fue realizada por personal capacitado a tal fin, con la obtención previa del consentimiento informado, que se realizó en forma verbal. En todos los casos, se constató la comprensión de la encuesta y sus alcances y la participación en el trabajo. ${ }^{7}$

El período analizado fue entre el 1 de febrero y el 31 de julio de 2015 (6 meses).

Los criterios de inclusión fueron madres sanas menores de 20 años, que estuvieran internadas con sus hijos menores de 1 año y que accedieran a responder la encuesta. Se las dividió en dos grupos: el grupo 1, de $<17$ años (adolescencia temprana-media), y el grupo 2, de $\geq 17$ años (adolescencia tardía).

Se midieron las siguientes variables: número de embarazos (uno o más); nacionalidad (argentina y otras); lugar de residencia (Ciudad Autónoma de Buenos Aires -CABA-, provincia de Buenos Aires y otras); abandono de la escuela de la madre durante el embarazo o después de él; edad de inicio de relaciones sexuales (IRS); continuidad en relación de pareja con el padre del niño; edad del padre del niño; repetición intergeneracional de embarazo adolescente; cobro de subsidios (Asignación Universal por Embarazo y por Hijo, Asignación por Embarazo para Protección Social); número de controles prenatales (de 1 a 4-5 o más); presencia de enfermedades y/o internaciones durante el embarazo; asistencia a controles puerperales; consumo de ácido fólico/hierro durante el embarazo; inicio de anticoncepción en el puerperio; conocimiento de las infecciones de transmisión sexual (ITS); edad gestacional y peso de nacimiento del niño internado; edad del niño al momento de realización de la encuesta; motivo actual de internación del niño.

Para la variable edad gestacional al nacer, se establecieron como prematuros aquellos niños con menos de 37 semanas por Capurro y, para la variable peso al nacer, se consideraron recién nacidos de muy bajo peso (RNMBP) los que hubieran tenido menos de 1500 gramos.

La repetición intergeneracional de embarazo adolescente es el fenómeno por el que una madre adolescente es hija de una mujer que también fue madre adolescente.

\section{Plan de análisis estadístico}

Los datos se registraron y trasladaron al software Microsoft Excel 2010. Las variables continuas se resumieron con medidas centrales y de dispersión acordes a su distribución. Las variables categóricas se resumieron con proporciones e índice de confianza del $95 \%$. Para realizar el análisis estadístico, se utilizó el software Stata 12.

El protocolo de investigación fue aprobado para su desarrollo en el Hospital, luego de cumplimentar el circuito de evaluación institucional que coordinaba la Dirección Asociada de Docencia e Investigación del Hospital Garrahan, que incluyó la aprobación de la Coordinación de Etica en el mes de septiembre de 2014. 


\section{RESULTADOS}

Durante el período estudiado, se internaron 763 madres adolescentes. De ellas, 227 cumplían los criterios y accedieron a participar.

La media de edad del grupo 1 fue de 15 años y 4 meses (DE 0.84) y la del grupo 2, de 18 años y 7 meses (DE 1.11) (Tabla 1).

Las madres del grupo 2 tenían un porcentaje mayor de 2 o más hijos: 21 (21\%, IC 95\%: 0,12$0,29)$ vs. 54 (42\%, IC 95\%: 0,33-0,51).

El inicio de relaciones sexuales fue significativamente más precoz entre las que fueron madres durante la adolescencia tempranamedia. Un 67\% (67) de ellas iniciaron a los 14 años o antes vs. el $51 \%$ (65) de las que fueron madres adolescentes tardías ( $\mathrm{p} 0,016$ ).

La continuidad en la relación de pareja con el padre del niño se vio con mayor frecuencia entre las adolescentes mayores al compararlas con las adolescentes tempranas-tardías $-89(70 \%)$ vs. $53(53 \%)$; p 0,008-.

Aquellas que no convivían con su pareja lo hacían con sus padres. Lo mismo ocurrió en relación con el cobro de subsidios, que fue significativamente mayor entre las adolescentes con edad $\geq 17$ años $-47(47 \%)$ vs. $78(61 \%)$; p 0,030-.

En ambos grupos, más de las dos terceras partes de las adolescentes eran hijas de madres adolescentes, y se repitió la historia generacional de embarazo adolescente $-76(76 \%)$ vs. $77(61 \%)$; p 0,015-.

Luego del embarazo, las $<17$ años usaban menos métodos anticonceptivos $-35(35 \%)$ vs.
$70(55 \%)$ - en comparación con las $\geq 17$ años (p 0,003) (Tabla 2).

En cuanto a la edad promedio del niño al momento de realización de la encuesta, fue, para el primer grupo, de 5,03 meses (IC 95\%: 4,50-5,55) y, para el segundo, de 7,24 meses (IC 95\%: 6,82-7,66).

Las infecciones, en especial la sepsis $-36(36 \%)$ vs. $32(25 \%)$-, fueron la patología predominante como motivo de internación del niño, seguidas de cardiopatías congénitas -17 (17\%) vs. $10(8 \%)$ - y patologías neurológicas $-16(16 \%)$ vs. $13(10 \%)-$ en ambos grupos, respectivamente. En 31 (31\%) y $72(57 \%)$ de ambos grupos, fueron otras causas las que motivaron la internación.

Los $\mathrm{RN} \leq 37$ semanas en madres menores de 17 años constituyeron el 37\% $(n=37)$ (IC 95\%: $27-46)$ y, en las $\geq 17$ años, fueron el $34 \%(n=43)$ (IC 95\%: 25,42), sin diferencias significativas.

En referencia al peso de nacimiento, se constató $12 \%(n=12$; IC 95\%: 5-18) de RNMBP en el grupo de madres menores de 17 años y $2 \%$ $(n=3$; IC 95\%: 0-5) en las $\geq 17$ años ( $p$ 0,004).

\section{DISCUSIÓN}

Se ha descrito en la literatura una asociación entre embarazo en etapas tempranas y mayor riesgo materno-fetal, dependencia económica, falta de consolidación de la pareja, violencia sexual y riesgo social. ${ }^{8}$

Gortzak-Uzan describe una frecuencia 12 veces mayor de RNMBP en embarazos de adolescentes tempranas. ${ }^{9,10}$ En concordancia con la literatura, se halló una mayor incidencia de RNMBP en el grupo de las madres menores.

TABLA 1. Características demográficas, sociales y familiares de las madres

\begin{tabular}{|c|c|c|c|}
\hline & Menores de 17 años $(n=100)$ & $\geq 17$ años $(n=127)$ & Valor de $p$ \\
\hline Media de la edad materna (DE) & 15 años y 4 meses $(0,84)$ & 18 años y 7 meses $(1,11)$ & \\
\hline 1 hijo & $79 \%(70-87)$ & $57 \%(48-66)$ & \\
\hline 2 o más hijos & $21 \%(12-29)$ & $43 \%(33-51)$ & \\
\hline \multicolumn{4}{|l|}{ Nacionalidad } \\
\hline Argentina & $94 \%(89-98)$ & $88 \%(82-93)$ & \\
\hline Otras & $6 \%(1,2-10)$ & $12 \%(6-17)$ & \\
\hline \multicolumn{4}{|l|}{ Domicilio } \\
\hline CABA & $24 \%(15-32)$ & $13 \%(7-19)$ & \\
\hline Conurbano & $63 \%(53-72)$ & $69 \%(60-76)$ & \\
\hline Otras provincias & $13 \%(6-19)$ & $18 \%(11-24)$ & \\
\hline \multicolumn{4}{|l|}{ Abandono de la escuela durante } \\
\hline el embarazo o después de él & $68 \%(58-77)$ & $58 \%(49-66)$ & \\
\hline \multicolumn{4}{|l|}{ IRS } \\
\hline 14 años o antes & $67 \%(57-76)$ & $51 \%(42-59)$ & \\
\hline Luego de los 15 años & $33 \%(23-42)$ & $49 \%(40-57)$ & 0,016 \\
\hline \multicolumn{4}{|l|}{ Continuidad en la relación de pareja } \\
\hline con el padre del niño & $53 \%(43-62)$ & $70 \%(62-78)$ & 0,008 \\
\hline \multicolumn{2}{|l|}{ Repetición intergeneracional de } & $61 \%(52-69)$ & 0,015 \\
\hline Cobro de subsidio & $47 \%(37-56)$ & $61 \%(52-69)$ & 0,030 \\
\hline
\end{tabular}

DE: desvío estándar; CABA: Ciudad Autónoma de Buenos Aires; IRS: inicio de relaciones sexuales. 
La prevalencia de prematurez fue elevada también en las madres de menor edad, al igual que en la literatura nacional e internacional, sin evidenciarse relevancia clínica entre ambos grupos. ${ }^{11}$

La edad temprana de inicio de relaciones sexuales se asocia frecuentemente como factor de riesgo para embarazo adolescente. Nuestro trabajo describe una edad de inicio por debajo del promedio descrito en los últimos trabajos nacionales (15,6 años). ${ }^{12}$

Las estadísticas nacionales informan que cerca del $85 \%$ de las embarazadas adolescentes tienen entre 3 y 9 consultas prenatales; en concordancia con esto, nuestras entrevistadas presentaron 5 controles en el embarazo (en ambos grupos), lo que resultaría adecuado.

En lo referente al abordaje posembarazo, se evidenció una baja asistencia a los controles posparto y deficiente anticoncepción en el puerperio, dato que coincidió con otros trabajos nacionales. ${ }^{13}$ Fue muy llamativa la tasa de repetición de embarazo adolescente en ambos grupos. En particular, en menores de 17 años, con $21 \%$. Sería importante reflexionar acerca de cuáles son las barreras que determinan este fenómeno, considerando la vigencia de la Ley Nacional $\mathrm{N}^{\mathrm{o}} 25673$ (provisión gratuita y universal de métodos anticonceptivos).${ }^{14}$ Es notoria la falta de información sobre las ITS, que deberían pesquisarse e informarse en forma sistemática en cualquier consulta.

Existen reportes que describen la existencia de una fuerte asociación entre embarazo adolescente en una generación y en la siguiente, situación que se evidencia en nuestro trabajo. Su alta frecuencia podría relacionarse con la transmisión de experiencias madre-hija, en las que se naturaliza la maternidad adolescente. ${ }^{15}$

En relación con las madres que mantenían su pareja durante el embarazo y después de él, nuestros resultados coinciden con la literatura, en la que se expone que muchos embarazos, aunque no planificados, se dan en el contexto de pareja y que la mayoría de los padres que no conviven no aportan al sustento de sus hijos. Las madres o abuelas de las adolescentes son las que desempeñan un rol en lo económico como en la crianza. ${ }^{16}$

Se evidencia una alta tasa de deserción escolar durante el embarazo con cifras mayores al promedio nacional. ${ }^{17}$ La mayoría de las adolescentes madres suelen dejar la escuela antes de la ocurrencia del embarazo, lo que duplica las posibilidades de ser madres en comparación con las que asisten. Asimismo, quienes concurren embarazadas abandonan con frecuencia. Esta situación reduce las herramientas para el futuro y favorece la marginalización y los trabajos precarios. Otro factor que profundiza la vulnerabilidad es la falta de acceso a los subsidios. En nuestro trabajo, las madres menores cobraron menos subsidios.

Las instituciones que cuentan con servicios diferenciados de atención de adolescentes tienen mayor posibilidad de aplicar un abordaje integral y un trato basado en el cumplimiento de sus derechos. Con el fin de reducir el embarazo adolescente, se deben priorizar las acciones que mejoren su detección oportuna, incluir en forma sistemática la pesquisa de situaciones de abuso y conformar equipos especializados que aseguren confidencialidad, privacidad, escucha e integralidad. Asimismo, es fundamental el acompañamiento pre- y posparto y la ampliación de políticas y acciones que contribuyan a promover la escolaridad y fomentar la educación sexual en todos los niveles.

Son necesarias evaluaciones para ir identificando limitaciones y desafíos en los diferentes niveles en los que se van implementando los programas y acciones. ${ }^{18}$

Este trabajo nos permitió aprovechar la internación para evitar "otra oportunidad

TABLA 2. Características del embarazo, finalización del embarazo y puerperio

\begin{tabular}{|c|c|c|c|}
\hline & Menores de 17 años $(n=100)$ & $\geq 17$ años $(n=127)$ & Valor de $p$ \\
\hline \multicolumn{4}{|l|}{ Control prenatal } \\
\hline 5 controles o más & $65 \%(55-74)$ & $68 \%(59-75)$ & \\
\hline De 1 a 4 controles & $35 \%(25-44)$ & $32 \%(24-40)$ & \\
\hline Enfermedades durante el embarazo & $37 \%(27-46)$ & $30 \%(21-37)$ & \\
\hline Internaciones durante el embarazo & $15 \%(7-22)$ & $10 \%(4-15)$ & \\
\hline Controles posparto & $38 \%(28-47)$ & $47 \%(38-56)$ & \\
\hline \multicolumn{4}{|l|}{ Ácido fólico suplementario durante } \\
\hline los primeros 3 meses de embarazo & $33 \%(23-42)$ & $44 \%(35-52)$ & \\
\hline $\begin{array}{l}\text { Hierro suplementario en la última } \\
\text { etapa del embarazo }\end{array}$ & $63 \%(53-72)$ & $74 \%(66-81)$ & \\
\hline $\begin{array}{l}\text { Utilización de métodos anticonceptivos } \\
\text { posterior al parto }\end{array}$ & $35 \%(25-44)$ & $55 \%(46-63)$ & 0,003 \\
\hline Conocimiento de ITS & $46 \%(36-55)$ & $54 \%(45-63)$ & \\
\hline
\end{tabular}

ITS: infecciones de transmisión sexual. 
perdida" y brindarles lo siguiente: 1 . educación, información sobre prevención y derechos sexuales y reproductivos; 2 . asistencia por médicos especialistas. A su vez, se ponderó el fomento del autocuidado de estas adolescentes y el de sus pares, y se garantizó el acceso a servicios de calidad.

Cabe destacar que nuestro Hospital se comporta como centro de referencia para distintos tipos de patologías graves y complejas, como cardiopatías congénitas, gastrosquisis, afecciones neuroquirúrgicas, etc., y nos impide sacar conclusiones extrapolables a la población general.

Otro sesgo podría estar determinado por la selección por conveniencia de la muestra. Sin embargo, se pueden describir las características de la población observada en el presente estudio y permitirnos algunas reflexiones.

\section{CONCLUSIONES}

Las adolescentes de edad mayor de 17 años o igual tuvieron significativamente mayor cantidad de hijos, cobraron más subsidios y tuvieron mayor nivel de convivencia con el padre de sus hijos en comparación con las adolescentes tempranas-medias.

Ambos grupos presentaron alta tasa de prematurez, pero las madres en la adolescencia temprana-media tuvieron más RNMBP. Este último grupo también presentó mayor porcentaje de inicio de relaciones sexuales antes de los 14 años y una escasa utilización de métodos anticonceptivos en el puerperio.

La deserción escolar fue un factor común en las dos poblaciones.

Del total de madres adolescentes, la historia intergeneracional de embarazo adolescente se repitió en más de dos tercios, en especial, en las menores de 17. También se evidenció un bajo número de controles posparto, lo que las exponía a nuevos embarazos.

\section{Agradecimientos}

A las Licenciadas en Enfermería Norma Barraza, Mirta Durán y María Ester Velázquez por la ayuda en la recolección de los datos.

A la Dras. Carolina Cernadas y Paula Weissbrod por su asesoramiento y orientación en el análisis estadístico de los datos.

\section{REFERENCIAS}

1. Organización Mundial de la Salud. Salud para los adolescentes del mundo. Una segunda oportunidad en la segunda década. Organización Mundial de la Salud. Ginebra, 2014. [Acceso: 31 de octubre de 2017]. Disponible en http: / / apps.who.int/ adolescent/second-decade/files / WHO_FWC_MCA_14.05_spa.pdf.

2. UNICEF. Embarazoy Maternidad en adolescentes menores de 15 años. Hallazgos y desafíos para las políticas públicas. Argentina. 2017. [Acceso:31 de octubre de2017]. Disponible en:https: / / www.unicef.org/argentina/spanish/SALUD-
maternidadAdolescenteFinal.pdf.

3. Alianza Argentina para la Salud de la Madre, Recién Nacido y Niño (ASUMEN). En nuestro país, cada 3 horas, una niña da a luz y se convierte en madre de otro niño o niña. Buenos Aires, Argentina. Mayo de 2014. [Acceso: 7 de marzo de 2017]. Disponible en: https: / / www.unicef. org/argentina/spanish / ASUMEN_ALERTA_embarazo_ menores_15_Mayo2014.pdf.

4. Argentina. Ministerio de Salud. Salud en la adolescencia. Boletín Virtual. 2016;3(10). [Acceso: 7 de marzo de 2017]. Disponible en: http:/ / www.msal.gob.ar/municipios / images / stories / 4-recursos / pdf/boletin_virtual-10-salud_ integral_adolescencia.pdf.

5. Argentina. Ministerio de Salud. CONAPRIS-CEDES. El embarazo en la adolescencia: diagnóstico para reorientar las políticas y programas de salud. Informe final. Buenos Aires, 2004. [Acceso: 7 de marzo de 2017]. Disponible en: http: / / www.cedes.org.ar/Publicaciones / IF / 2004/1698.pdf.

6. Gaete V. Desarrollo psicosocial del adolescente. Rev Chil Pediatr 2015;86(6):436-43.

7. Ley Nacional Argentina N.o 26529. Derechos del paciente en su relación con los profesionales e instituciones de la salud. Buenos Aires, 19 de noviembre de 2009. [Acceso: 7 de marzo de 2017]. Disponible en: http: / / www.uba.ar/ archivos_secyt/image/Ley\%2026529.pdf.

8. Gómez P, Molina R, Zamberlin N. Factores relacionados con el embarazo y la maternidad en menores de 15 años en américa latina y el caribe. Lima: FLASOG; 2011:1730. [Acceso: 31 de octubre de 2017]. Disponible en: http: / / www.unal.edu.co/ bioetica/documentos / 2011/ maternidad.pdf.

9. Gortzak-Uzan L, Hallak M, Press F, et al. Teenage pregnancy: risk factors for adverse perinatal outcome. $J$ Matern Fetal Med 2001;10(6):393-7.

10. Kushwaha K, Rai Ak, Rathi Ak, et al. Pregnancies in adolescents: fetal, neonatal and maternal outcome. Indian Pediatr 1993;30(4):501-5.

11. Cluet de Rodríguez I, Rossell-Pineda M, Alvarez de Acosta $\mathrm{T}$, et al. Factores de riesgo asociados a la prematuridad en recién nacidos de madres adolescentes. Rev Obstet Ginecol Venez 2013;73(3):157-70.

12. Oiserovich $S$, Daldevich D, Salvo M, et al. Investigación exploratoria sobre características de crecimiento desarrollo y cuidados de la salud sexual y reproductiva en población adolescente. Sociedad Argentina de Ginecología Infanto Juvenil. 2010. [Acceso: 7 de marzo de 2017]. Disponible en: conders. feim.org.ar/pdf/sagij.pdf.

13. Gogna M, Binstock $G$, Fernández $S$, et al. Adolescent pregnancy in Argentina: evidence-based recomendations for public policies. Reprod Health Matters 2008;16(31):192-201.

14. Ley Nacional Argentina N.o 25673. Programa Nacional de Salud y Procreación Responsable. Buenos Aires, 26 de mayo de 2003. [Acceso: 7 de marzo de 2017]. Disponible en: http: / / www.msal.gob.ar/saludsexual/ley.php.

15. González E, Leal I, Molina T, et al. Patrón intergeneracional del embarazo adolescente en las hijas de una cohorte de mujeres que controlaron su primer embarazo en un centro integral para adolescentes embarazadas. Rev Chil Obstet Ginecol 2013;78(4):282-9.

16. Carricondo M, Bleisen R. Embarazo adolescente: aportes desde el trabajo social a una problematica de larga trayectoria. Caso: Comunidad de Palmira. Año 2013. [Tesis] Mendoza: Universidad Nacional de Cuyo; 2014. [Acceso: 3 de noviembre de 2017]. Disponible en: http: / / bdigital. uncu.edu.ar/objetos_digitales / 6564/ tesis-fcpys-ts-2014bleisen-carricondo.p $\bar{d} f$.

17. La UNESCO y la educación. París: UNESCO;2009. [Acceso: 7 demarzo de 2017]. Disponibleen: http: / / unesdoc.unesco. org/images/0018/001849/184967s.pdf.

18. Prevención y reducción del embarazo no intencional en la población adolescente del Cono Sur. Un marco estratégico subregional para mejorar la implementación de la política local. Asunción; 2017. [Acceso: 3 de noviembre de 2017]. Disponible en: http: / / lac.unfpa.org/sites / default/files / pub-pdf $/$ marco $\% 20$ estrategico $\% 20$ castellano $\% 200 K \% 20$ 17bMayo_1.pdf. 
ANEXO

PROGRAMA DE ATENCIÓN DE MADRES ADOLESCENTES EN INTERNACIÓN HISTORIA CLÍNICA DE LAS MADRES ADOLESCENTES

FECHA:......................

Edad materna:............. Sector de internación:....

Madre de:...................................................

Motivo de internación del hijo-a:.....

Lugar de residencia: CABA $\square$ Conurbano $\square$ Otras provincias

Nacionalidad:

Convivientes:.

Abandono de la escolaridad durante el embarazo o después de él: $\quad$ Sí $\square$ No $\square$

Cobro de subsidio: Sí

No

Edad de su madre en su primer embarazo:.

Edad del papá del niño:.

Continuidad en la relación de pareja con el padre del niño: Sí $\square$ No

Edad del inicio de relaciones sexuales:.

Número de hijos:

Edad del primer embarazo:.........

Control de embarazo: $\quad$ Sí $\square$ No

Enfermedad o problema de salud en el embarazo:

Sí $\square$ No $\square$

¿Cuál?.

Internaciones en el embarazo: $\quad$ Sí $\square$ No $\square$ ¿Por qué?

Toma de ácido fólico: Sí $\square$ No $\square$

Toma de hierro: $\quad$ Sí $\square$ No $\square$ Durante el embarazo

Controles luego del parto: $\quad$ Sí $\square$ No $\square$

Uso de métodos anticonceptivos después del parto: Sí $\square$ No $\square$ ¿Cuál?.............

Edad gestacional de nacimiento del hijo/a:

Peso de nacimiento: 